\title{
Positive Solutions for a Fractional Boundary Value Problem with a Perturbation Term
}

\author{
Yumei Zou (iD) \\ Department of Statistics and Finance, Shandong University of Science and Technology, Qingdao 266590, China
}

Correspondence should be addressed to Yumei Zou; sdzouym@126.com

Received 9 April 2018; Accepted 23 May 2018; Published 7 August 2018

Academic Editor: Liguang Wang

Copyright (C) 2018 Yumei Zou. This is an open access article distributed under the Creative Commons Attribution License, which permits unrestricted use, distribution, and reproduction in any medium, provided the original work is properly cited.

We obtain some new upper and lower estimates for the Green's function associated with a fractional boundary value problem with a perturbation term. Criteria for the existence of positive solutions of the problem are then obtained based on it.

\section{Introduction}

In this paper, we are investigating the existence of positive solution for fractional differential equation with a perturbation term

$$
-D^{\alpha} x(t)+a(t) x(t)=f(t, x(t)), \quad t \in(0,1)
$$

with the boundary condition (BC)

$$
x(0)=x^{\prime}(0)=x^{\prime}(1)=0,
$$

where $2<\alpha<3, a \in C[0,1]$, and $f \in C([0,1] \times[0,+\infty), \mathbb{R})$. Here, $D^{\alpha} x$ is the standard Riemann-Liouville derivative of order $\alpha>0$ of a continuous function $x:(0, \infty) \rightarrow \mathbb{R}$ is given by

$$
\begin{array}{r}
D^{\alpha} x(t)=\frac{1}{\Gamma(n-\alpha)}\left(\frac{d}{d t}\right)^{n} \int_{0}^{t} \frac{x(s)}{(t-s)^{\alpha-n+1}} d s, \\
\quad n-1 \leq \alpha<n
\end{array}
$$

provided that the right-hand side is pointwise defined on $(0, \infty)$.

Spurred by the extensively applicability of fractional derivatives in a variety of mathematical models in science and engineering [1-3], the subject of fractional differential equations with boundary value problems, which emerged as a new branch of differential equations, have attracted a great deal of attention for decades. As a small sampling of recent development, we refer the reader to [4-14]. When one seeks the existence of solution of boundary value problems for fractional differential equations, the usual method is converted to a Fredholm integral equation and find the fixed points by using various techniques of nonlinear analysis such as Banach contraction map principle $[13,15]$, linear operator theory [16, 17], Leggett-Williams fixed point theorem $[12,18]$, Schauder fixed point theorem and Leray-Schauder nonlinear alternative theory [19], and Krasnosel'skii fixed point theorem [20]. It should be noted that the Green's functions play a vital role in the construction of an appropriate Fredholm integral equation. However, as a result of the unusual feature of the fractional calculus, the investigation on the Green's functions for fractional boundary value problems is still in the initial stage. Recently, based on the spectral theory, the authors in [21] give an associated Green's function for BVP (1) (2) as series of functions. This idea was also used in [22-24].

In the next section, we will study some new sharper upper and lower estimates for the Green's function of BVP (1) (2) than the ones given in [21]. In Section 3, we employ the new estimate to obtain the existence of a positive solution of BVP (1) (2). The idea of this paper may trace to [21-27].

\section{Some New Upper and Lower Estimates for the Green's Function}

Firstly, we present the Green's function for BVP (1) (2) which is given in [21]. Let $G_{0}:[0,1] \times[0,1] \longrightarrow \mathbb{R}$ be defined by

$$
G_{0}(t, s)
$$




$$
= \begin{cases}\frac{t^{\alpha-1}(1-s)^{\alpha-2}-(t-s)^{\alpha-1}}{\Gamma(\alpha)}, & 0 \leq s \leq t \leq 1, \\ \frac{t^{\alpha-1}(1-s)^{\alpha-2}}{\Gamma(\alpha)}, & 0 \leq t \leq s \leq 1 .\end{cases}
$$

Lemma 2. If $M<1$ holds, then $r(T)<1$.

Proof. By Lemma 1 , for any $x \in C[0,1]$, we have

$$
\begin{aligned}
|(T x)(t)| & \leq \int_{0}^{1} G_{0}(t, s)|a(s) x(s)| d s \\
& \leq \frac{t^{\alpha-1} A\|x\|}{\Gamma(\alpha)} \int_{0}^{1}(1-s)^{\alpha-2} d s \\
& =\frac{A\|x\|}{(\alpha-1) \Gamma(\alpha)} t^{\alpha-1} .
\end{aligned}
$$

Hence, we conclude that

$$
\begin{aligned}
& \left|\left(T^{2} x\right)(t)\right| \leq \int_{0}^{1} G_{0}(t, s)|a(s)(T x)(s)| d s \\
& \quad \leq \frac{A\|x\|}{(\alpha-1) \Gamma(\alpha)} \int_{0}^{1} \frac{t^{\alpha-1}|a(s)|(1-s)^{\alpha-2}}{\Gamma(\alpha)} s^{\alpha-1} d s \\
& \quad=\frac{A M\|x\|}{(\alpha-1) \Gamma(\alpha)} t^{\alpha-1} .
\end{aligned}
$$

By induction, one has

$$
\left|\left(T^{n} x\right)(t)\right| \leq \frac{A M^{n-1}\|x\|}{(\alpha-1) \Gamma(\alpha)} t^{\alpha-1}
$$

which implies that

$$
\left\|T^{n}\right\| \leq \frac{A M^{n-1}}{(\alpha-1) \Gamma(\alpha)} .
$$

Note that $M<1$. Then by the Gelfand formula, we get

$$
r(T)=\lim _{n \longrightarrow+\infty} \sqrt[n]{\left\|T^{n}\right\|} \leq M<1
$$

$$
G(t, s)=\sum_{n=0}^{+\infty}(-1)^{n} G_{n}(t, s)
$$

It follows from Theorem 2.1 in [21] that the function $G(t, s)$ defined by (6) as a series of functions that converge uniformly is the Green's function for BVP (1) (2) if $A<(\alpha-$ 1) $\Gamma(\alpha+1)$ holds. Furthermore, the function $G(t, s)$ satisfies the following property:

$$
(1-\delta) G_{0}(t, s) \leq G(t, s) \leq(1+\delta) G_{0}(t, s),
$$

$$
t, s \in[0,1]
$$

provided $A<(\alpha-1) \Gamma(\alpha+1)(\alpha+1)^{-1}$, where $\delta=\alpha A /((\alpha-$ 1) $\Gamma(\alpha+1)-A)<1$.

The uniform convergence of (6) follows from the fact that $\|T\|<1$, where the operator $T$ is defined by the following form:

$$
(T x)(t)=\int_{0}^{1} G_{0}(t, s) a(s) x(s) d s, \quad x \in C[0,1] .
$$

Indeed, the uniform convergence of (6) can be obtained by $r(T)<1$ (see $[21,29]$ ), where $r(T)$ is the spectral radius of $T$.
Lemma 3. If $M+M_{1}<1$ holds, then for any $t, s \in[0,1]$,

$$
\begin{aligned}
\frac{1-M-M_{1}}{1-M} G_{0}(t, s) & \leq G(t, s) \\
& \leq \frac{1-M+M_{1}}{1-M} G_{0}(t, s) .
\end{aligned}
$$

Proof. By Lemma 1 , for $n \geq 1$ and $t, s \in[0,1]$, we have

$$
\begin{aligned}
& \left|G_{n}(t, s)\right| \leq \int_{0}^{1} \cdots \int_{0}^{1}\left|a\left(r_{1}\right)\right| G_{0}\left(t, r_{1}\right) \cdot\left|a\left(r_{2}\right)\right| \\
& \cdot G_{0}\left(r_{1}, r_{2}\right) \cdots\left|a\left(r_{n}\right)\right| G_{0}\left(r_{n-1}, r_{n}\right) \\
& \cdot G_{0}\left(r_{n}, s\right) d r_{1} \cdots d r_{n} \\
& \quad \leq \int_{0}^{1} \cdots \int_{0}^{1}\left|a\left(r_{1}\right)\right| \frac{t^{\alpha-1}\left(1-r_{1}\right)^{\alpha-2}}{\Gamma(\alpha)} \cdot\left|a\left(r_{2}\right)\right| \\
& \cdot \frac{r_{1}^{\alpha-1}\left(1-r_{2}\right)^{\alpha-2}}{\Gamma(\alpha)} \cdots \frac{\left|a\left(r_{n}\right)\right| r_{n-1}{ }^{\alpha-1}\left(1-r_{n}\right)^{\alpha-2}}{\Gamma(\alpha)}
\end{aligned}
$$




$$
\begin{aligned}
& \cdot \frac{s(1-s)^{\alpha-2}}{\Gamma(\alpha)} d r_{1} \cdots d r_{n}=\frac{t^{\alpha-1} s(1-s)^{\alpha-2}}{\Gamma(\alpha)} \\
& \cdot \int_{0}^{1} \frac{\left|a\left(r_{1}\right)\right| r_{1}^{\alpha-1}\left(1-r_{1}\right)^{\alpha-2}}{\Gamma(\alpha)} d r_{1} \\
& \cdot \int_{0}^{1} \frac{\left|a\left(r_{2}\right)\right| r_{2}^{\alpha-1}\left(1-r_{2}\right)^{\alpha-2}}{\Gamma(\alpha)} d r_{2} \\
& \ldots \int_{0}^{1} \frac{\left|a\left(r_{n-1}\right)\right| r_{n-1}^{\alpha-1}\left(1-r_{n-1}\right)^{\alpha-2}}{\Gamma(\alpha)} d r_{n-1} \\
& \cdot \int_{0}^{1} \frac{\left|a\left(r_{n}\right)\right|\left(1-r_{n}\right)^{\alpha-2}}{\Gamma(\alpha)} d r_{n}=M_{1} M^{n-1} \\
& \cdot \frac{t^{\alpha-1} s(1-s)^{\alpha-2}}{\Gamma(\alpha)} \leq M_{1} M^{n-1} G_{0}(t, s),
\end{aligned}
$$$$
t, s \in[0,1]
$$

Then introducing the above inequality into (6) can lead to

$$
\begin{aligned}
\left|\sum_{n=1}^{+\infty}(-1)^{n} G_{n}(t, s)\right| & \leq \sum_{n=1}^{+\infty}\left|G_{n}(t, s)\right| \\
& \leq \sum_{n=1}^{+\infty} M_{1} M^{n-1} G_{0}(t, s) \\
& =\frac{M_{1}}{1-M} G_{0}(t, s), \quad t, s \in[0,1] .
\end{aligned}
$$

It is easy to verify that if $M+M_{1}<1$, then $M_{1} /(1-M)<1$. Therefore, (14) follows from (6) and (16).

Similar to the proof of Lemmas 2 and 3, we can obtain the following results.

Lemma 4. If $A<\Gamma(2 \alpha-1) / \Gamma(\alpha-1)$ holds, then $r(T)<1$.

Lemma 5. If $A /(\alpha-1) \Gamma(\alpha)+A \Gamma(\alpha-1) / \Gamma(2 \alpha-1)<1$ holds, then for any $t, s \in[0,1]$,

$$
(1-\gamma) G_{0}(t, s) \leq G(t, s) \leq(1+\gamma) G_{0}(t, s),
$$

where $\gamma=(A /(\alpha-1) \Gamma(\alpha))(1-A \Gamma(\alpha-1) / \Gamma(2 \alpha-1))^{-1}$.

By the properties of definite integral and $\alpha \in(2,3)$, we assert that

$$
\int_{0}^{1} \frac{\tau^{\alpha-1}(1-\tau)^{\alpha-2}}{\Gamma(\alpha)} d \tau<\int_{0}^{1} \frac{\tau(1-\tau)^{\alpha-2}}{\Gamma(\alpha)} d \tau,
$$

that is

$$
\frac{\Gamma(\alpha-1)}{\Gamma(2 \alpha-1)}<\frac{1}{(\alpha-1) \Gamma(\alpha+1)} .
$$

Thus, we obtain that

$$
\begin{aligned}
\gamma & =\frac{A /(\alpha-1) \Gamma(\alpha)}{1-A \Gamma(\alpha-1) / \Gamma(2 \alpha-1)} \\
& <\frac{\alpha A /(\alpha-1) \Gamma(\alpha+1)}{1-A /(\alpha-1) \Gamma(\alpha+1)}=\delta .
\end{aligned}
$$

This means that Lemmas $2-5$ is more general and complements many known results.

Combining Lemmas 1 and 3, we obtain the following result.

Theorem 6. If $M+M_{1}<1$ holds. Then for any $t, s \in[0,1]$,

$$
\begin{aligned}
& \frac{1-M-M_{1}}{1-M} t^{\alpha-1} G_{0}(1, s) \leq G(t, s) \\
& \leq \frac{1-M+M_{1}}{1-M} G_{0}(1, s) .
\end{aligned}
$$

Theorem 7. If $x(t)$ satisfies the boundary conditions (2),

$$
-D^{\alpha} x(t)+a(t) x(t) \geq 0 .
$$

If $M+M_{1}<1$ holds, then

$$
x(t) \geq \frac{1-M-M_{1}}{1-M+M_{1}} t^{\alpha-1}\|x\|, \quad t \in[0,1] .
$$

\section{Existence Theorems}

In this section, we shall employ Theorem 6 to investigate the existence results for BVP (1) (2). Let $C[0,1]$ be the Banach space endowed with the maximum norm $\|x\|=$ $\max _{t \in[0,1]}|x(t)|$.

Theorem 8. Assume that there exist $c_{2}>c_{1}>0$ such that

$$
\inf _{x \in \Omega} \int_{0}^{1} G_{0}(1, s) f(s, x(s)) d s \geq \frac{c_{1}(1-M)}{1-M-M_{1}}
$$

and

$$
\sup _{x \in \Omega} \int_{0}^{1} G_{0}(1, s) f(s, x(s)) d s \leq \frac{c_{2}(1-M)}{1-M+M_{1}},
$$

where

$$
\Omega=\left\{x \in C[0,1]: c_{1} t^{\alpha-1} \leq x(t) \leq c_{2}, t \in[0,1]\right\} .
$$

Then BVP (1) (2) has at least one positive solution in $\Omega$.

Proof. Define an operator $T$ by

$$
(S x)(t)=\int_{0}^{1} G(t, s) f(s, x(s)) d s, \quad x \in C[0,1],
$$

where $G(t, s)$ is given by (6). Obviously, $x(t)$ is a solution of BVP (1)(2) if and only if $x \in C[0,1]$ is a fixed point of $S$. Moreover, we can show that $S: C[0,1] \longrightarrow C[0,1]$ is completely continuous. 
For any given $x \in \Omega$, by (24) and (25), we conclude that

$(S x)(t)$

$$
\begin{aligned}
& \geq \frac{1-M-M_{1}}{1-M} t^{\alpha-1} \inf _{x \in S} \int_{0}^{1} G_{0}(1, s) f(s, x(s)) d s \\
& \geq c_{1} t^{\alpha-1}
\end{aligned}
$$

and

$$
\begin{aligned}
(S x)(t) & \leq \frac{1-M+M_{1}}{1-M} \sup _{x \in S} \int_{0}^{1} G_{0}(1, s) f(s, x(s)) d s \\
& \leq c_{2} .
\end{aligned}
$$

Therefore, $S(\Omega) \subset \Omega$. By Schauder's fixed point theorem, $S$ has a fixed point $x$ in $\Omega$ which implies that BVP (1) (2) has at least one positive solution in $\Omega$.

The following corollaries are direct results of Theorem 8 .

Corollary 9. Assume that there exist $c_{2}>c_{1}>0$ such that for any $t \in[0,1], f(t, \cdot)$ is nondecreasing on $\left[0, c_{2}\right]$,

$$
\int_{0}^{1} G_{0}(1, s) f\left(s, c_{1} s^{\alpha-1}\right) d s \geq \frac{c_{1}(1-M)}{1-M-M_{1}}
$$

and

$$
\int_{0}^{1} G_{0}(1, s) f\left(s, c_{2}\right) d s \leq \frac{c_{2}(1-M)}{1-M+M_{1}} .
$$

Then BVP (1) (2) has at least one positive solution in $\Omega$.

Corollary 10. Assume that there exist $c_{2}>c_{1}>0$ such that for any $t \in[0,1], f(t, \cdot)$ is nonincreasing on $\left[0, c_{2}\right]$,

$$
\int_{0}^{1} G_{0}(1, s) f\left(s, c_{1} s^{\alpha-1}\right) d s \leq \frac{c_{2}(1-M)}{1-M+M_{1}}
$$

and

$$
\int_{0}^{1} G_{0}(1, s) f\left(s, c_{2}\right) d s \geq \frac{c_{1}(1-M)}{1-M-M_{1}} .
$$

Then BVP (1) (2) has at least one positive solution in $\Omega$.

Example 11. Consider the BVP

$$
\begin{array}{r}
-D^{5 / 2} x(t)+t(1-t) x(t)=\sqrt{x(t)}, \quad t \in(0,1), \\
x(0)=x^{\prime}(0)=x^{\prime}(1)=0 .
\end{array}
$$

After simple computation, we have $M=\Gamma(\alpha+1) / \Gamma(2 \alpha+1)=$ $\sqrt{\pi} / 64, M_{1}=1 / \Gamma(\alpha+2)=16 \sqrt{\pi} / 105 \pi$.

Let $f(t, x)=\sqrt{x}$. It is easy to see that (30) and (31) hold when $c_{1}$ is small enough and $c_{2}$ is large enough. Then, by Corollary 9, BVP (34) has at least one solution.

\section{Data Availability}

The data used to support the findings of this study are included within the article.

\section{Conflicts of Interest}

The authors declare that there are no conflicts of interest regarding the publication of this paper.

\section{Acknowledgments}

The research is supported by the National Natural Science Foundation of China (11371221 and 51774197) and Shandong Natural Science Foundation (ZR2018MA011).

\section{References}

[1] A. A. Kilbas, H. M. Srivastava, and J. J. Trujillo, Theory and Applications of Fractional Differential Equations, New York, NY, USA, Elsevier, 2006.

[2] C. Lu, C. Fu, and H. Yang, "Time-fractional generalized Boussinesq equation for Rossby solitary waves with dissipation effect in stratified fluid and conservation laws as well as exact solutions," Applied Matheamtics and Computation, vol. 327, pp. 104-116, 2018.

[3] H. W. Yang, X. Chen, M. Guo, and Y. D. Chen, "A new ZK-BO equation for three-dimensional algebraic Rossby solitary waves and its solution as well as fission property," Nonlinear Dynamics, vol. 91, no. 3, pp. 1-14, 2017.

[4] Z. Bai, SH. Zhang, S. Sun, and Ch. Yin, "Monotone iterative method for a class of fractional differential equations," Electronic Journal of Differential Equations, vol. 2016, no. 06, pp. 1-8, 2016.

[5] Z. Bai, "On positive solutions of a nonlocal fractional boundary value problem," Nonlinear Analysis: Theory, Methods \& Applications, vol. 72, no. 2, pp. 916-924, 2010.

[6] A. Cabada and Z. Hamdi, "Nonlinear fractional differential equations with integral boundary value conditions," Applied Mathematics and Computation, vol. 228, pp. 251-257, 2014.

[7] Y. Cui, W. Ma, X. Wang, and X. Su, "Uniqueness theorem of differential system with coupled integral boundary conditions," Electronic Journal of Qualitative Theory of Differential Equations, no. 9, pp. 1-10, 2018.

[8] X. Hao, "Positive solution for singular fractional differential equations involving derivatives," Advances in Difference Equations, vol. 2016, article no. 139, 2016.

[9] X. Hao, H. Wang, L. Liu, and Y. Cui, "Positive solutions for a system of nonlinear fractional nonlocal boundary value problems with parameters and $p$-Laplacian operator," Boundary Value Problems, vol. 2017, article no. 182, 2017.

[10] L. Liu, F. Sun, X. Zhang, and Y. Wu, "Bifurcation analysis for a singular differential system with two parameters via to topological degree theory," Lithuanian Association of Nonlinear Analysts. Nonlinear Analysis: Modelling and Control, vol. 22, no. 1, pp. 31-50, 2017.

[11] F. Yan, M. Zuo, and X. Hao, "Positive solution for a fractional singular boundary value problem with p-Laplacian operator," Boundary Value Problems, article no. 51, 10 pages, 2018.

[12] X. Zhang and Q. Zhong, "Triple positive solutions for nonlocal fractional differential equations with singularities both on time and space variables," Applied Mathematics Letters, vol. 80, pp. 12-19, 2018. 
[13] Y. Zou and G. He, "A fixed point theorem for systems of nonlinear operator equations and applications to ( $p 1, p 2)$ Laplacian system," Mediterranean Journal of Mathematics, vol. 15, article no. 74, 11 pages, 2018.

[14] M. Zuo, X. Hao, L. Liu, and Y. Cui, "Existence results for impulsive fractional integro-differential equation of mixed type with constant coefficient and antiperiodic boundary conditions," Boundary Value Problems, vol. 2017, article no. 161, 2017.

[15] X. Zhang and Q. Zhong, "Uniqueness of solution for higherorder fractional differential equations with conjugate type integral conditions," Fractional Calculus and Applied Analysis, vol. 20, no. 6, pp. 1471-1484, 2017.

[16] Y. Cui, "Uniqueness of solution for boundary value problems for fractional differential equations," Applied Mathematics Letters, vol. 51, pp. 48-54, 2016.

[17] Y. Cui, W. Ma, Q. Sun, and X. Su, "New uniqueness results for boundary value problem of fractional differential equation," Nonlinear Analysis: Modelling and Control, vol. 23, no. 1, pp. 3139, 2018.

[18] Qiao Sun, Hongwei Ji, and Yujun Cui, "Positive Solutions for Boundary Value Problems of Fractional Differential Equation with Integral Boundary Conditions," Journal of Function Spaces, vol. 2018, Article ID 6461930, 6 pages, 2018.

[19] Tingting Qi, Yansheng Liu, and Yujun Cui, "Existence of Solutions for a Class of Coupled Fractional Differential Systems with Nonlocal Boundary Conditions," Journal of Function Spaces, vol. 2017, Article ID 6703860, 9 pages, 2017.

[20] X. Hao, M. Zuo, and L. Liu, "Multiple positive solutions for a system of impulsive integral boundary value problems with sign-changing nonlinearities," Applied Mathematics Letters, vol. 82, pp. 24-31, 2018.

[21] J. R. Graef, L. Kong, Q. Kong, and M. Wang, "On a fractional boundary value problem with a perturbation term," Journal of Applied Analysis and Computation, vol. 7, no. 1, pp. 57-66, 2017.

[22] J. R. Graef, L. Kong, Q. Kong, and M. Wang, "Fractional boundary value problems with integral boundary conditions," Applicable Analysis: An International Journal, vol. 92, no. 10, pp. 2008-2020, 2013.

[23] J. R. Graef, L. Kong, Q. Kong, and M. Wang, "Existence and uniqueness of solutions for a fractional boundary value problem with Dirichlet boundary condition," Electronic Journal of Qualitative Theory of Differential Equations, No. 55, 11 pages, 2013.

[24] J. R. Graef, L. Kong, Q. Kong, and M. Wang, "A fractional boundary value problem with dirichlet boundary condition," Communications in Applied Analysis, vol. 19, pp. 497-504, 2015.

[25] Y. Cui, Q. Sun, and X. Su, "Monotone iterative technique for nonlinear boundary value problems of fractional order $\mathrm{p} \in(2$, 3]," Advances in Difference Equations, vol. 2017, article no. 248, 2017.

[26] Y. Cui and Y. Zou, "Monotone iterative technique for $(k, n-$ k) conjugate boundary value problems," Electronic Journal of Qualitative Theory of Differential Equations, no. 69, pp. 1-11, 2015.

[27] J. R. Graef, L. Kong, and B. Yang, "Positive solutions for a fractional boundary value problem," Applied Mathematics Letters, vol. 56, pp. 49-55, 2016.

[28] Moustafa El-Shahed, "Positive Solutions for Boundary Value Problem of Nonlinear Fractional Differential Equation," Abstract and Applied Analysis, vol. 2007, Article ID 10368, 8 pages, 2007.
[29] E. Zeidler, Nonlinear Functional Analysis and its Application: Fixed Point-Theorems, Springer, 1986. 


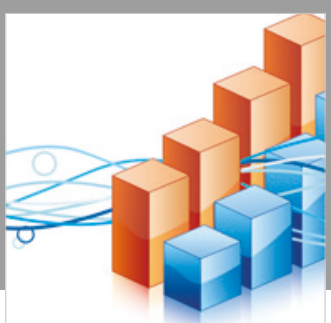

Advances in

Operations Research

\section{-n-m}
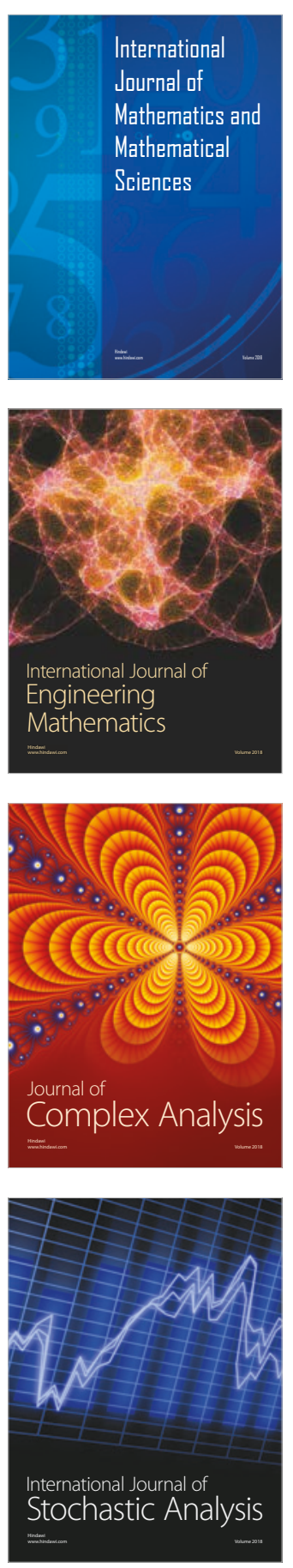
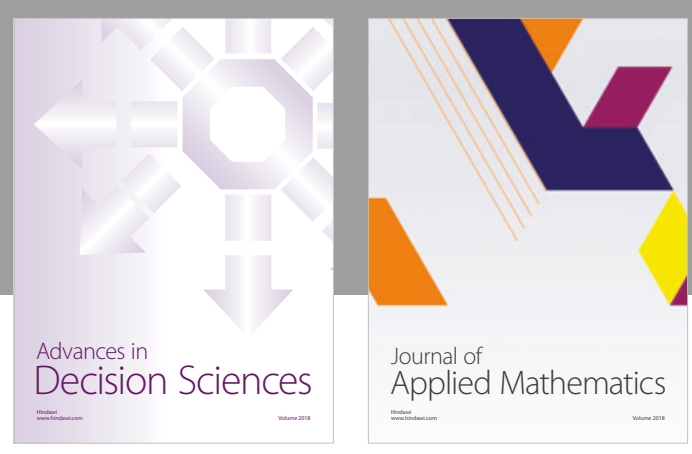

Journal of

Applied Mathematics
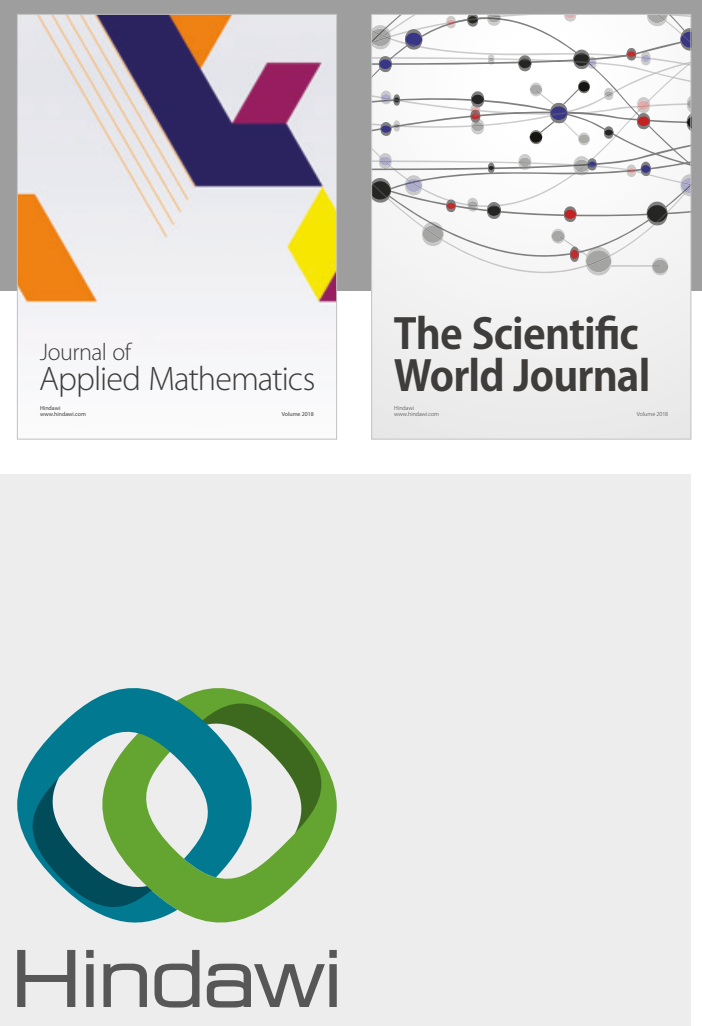

Submit your manuscripts at

www.hindawi.com

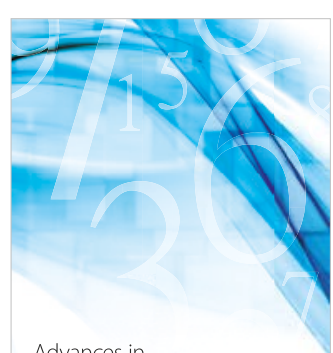

Advances in
Numerical Analysis
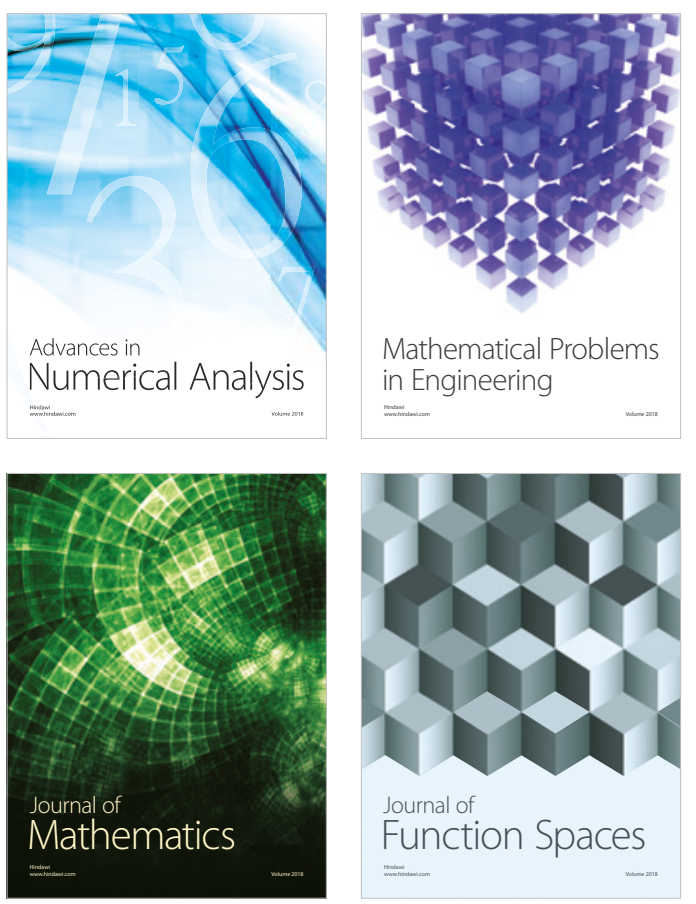

Mathematical Problems in Engineering

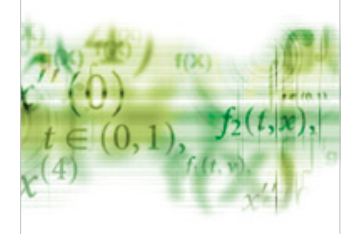

International Journal of

Differential Equations

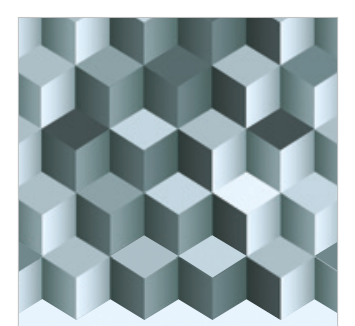

Journal of

Function Spaces

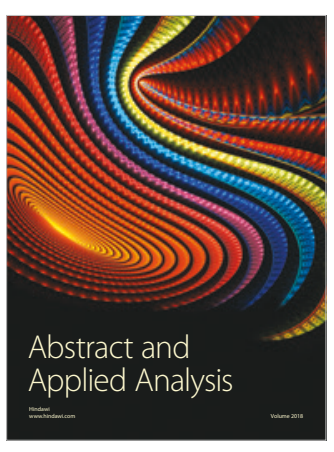

The Scientific

World Journal

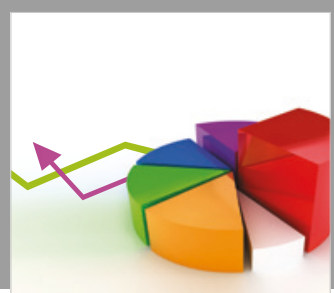

Journal of

Probability and Statistics
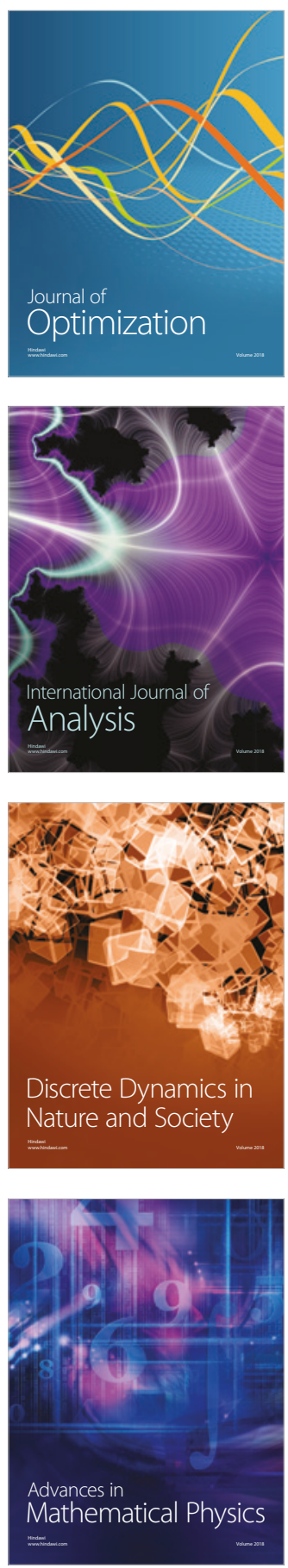